\title{
Advances in hematology - research that revolutionized patient care
}

\begin{abstract}
In the last decades, substantial strides have been made in the diagnosis, treatment, and prevention of blood diseases. The new drugs to be used in combination with cytostatic therapy have been developed, based on increased understanding of the biology of neoplasia. The diagnosis of several diseases is based exclusively on cytogenetic and molecular analysis which has become a part of routine diagnostic management. Moreover, molecular definition has allowed the introduction of therapy targeted at molecular change characteristic for a given disease. The introduction of novel agents for the treatment of hematological disorders has resulted in a great improvement in response rate and median survival.

The aim of this study is to show advances and possible future directions in the treatment of chosen hematological malignancies during the recent decades.
\end{abstract}

Keywords: chronic lymphocytic leukemia, immunochemotherapy, monoclonal antibodies, multiple myeloma, proteasome inhibitors, immunomodulatory drugs, chronic myeloid leukemia, tyrosine kinase inhibitors, bone marrow transplantation.

DOI: $10.1515 /$ pjph-2015-0019

\section{INTRODUCTION}

The concept of blood circulation was first introduced in 1628. Since then, there have been numerous advances in hematology and they had an immensely positive impact on the lives of patients with hematologic disorders and blazed a trail for advances in other fields. In the last 50 years alone, huge progress has been made in the research, treatment, and prevention of blood diseases. Past experience showed us that even some of the most basic research taking place today, has the potential to lead to promising life-saving treatments in the future.

The diagnosis of several diseases is based exclusively on cytogenetic and molecular analysis, which has become a part of the routine diagnostic management. Moreover, molecular definition has allowed the introduction of therapy targeted at molecular change characteristic for a given disease. The diagnosis of several diseases is based exclusively on cytogenetic and molecular analysis which has become a part of routine diagnostic management. The development of a specific treatment method for chronic myeloid leukemia (CML) is a remarkable story of scientific discovery proving that a precise understanding of the molecular cause of a cancer can lead to an effective and non-toxic "targeted" treatment. Prior to 1975, rapid progress had also been made in the use of chemotherapy for the treatment of lymphoma. Increasingly intense regimens caused an increase in toxicity, yet, without making any significant improvements in patient survival rates. Consequently, researchers started looking for a new approach, and it was clear that antibodies might provide the clues needed to diagnose and treat cancer. Antibodies are one way the immune system recognizes and destroys foreign invaders. Even though cancer cells are no foreign bodies, as they are part of the human body, some of their features can make them look foreign. Earlier, making cancer antibodies in a lab was challenging, since there were problems with finding the right antibodies, and making them in sufficient quantities with adequate purity.

In 1868, two researchers from Prussia and Italy made pioneering research and reported that bone marrow generates blood cells in mammals. This observation triggered intense efforts to replace malfunctioning marrow through the transplantation of blood-forming (hematopoietic) cells in humans. However, the development of reliable techniques to identify suitable donors, design potent antibiotics, and establish essential supportive care methods to successfully perform clinical hematopoietic cell transplantation (HCT) procedure took an entire century. Were it not for decades of intense preclinical animal research, this important accomplishment would not have been possible. This mainly pertains to graft rejection and the prevention of graft-versus-host disease (GVHD), a serious reaction caused by the donor's immune cells attacking the recipient's vital organs.

Today, we must ask ourselves about how many other potential new therapies are available and how can we be creative enough to bring them forward. The aim of this study was to show advances and possible future directions in the treatment of hematological malignancies during the recent 
decades. Only few of them are listed here, which is not to say that all the others are less important.

\section{Advances in the treatment of chronic lymphocytic leukemia}

In the past few years, we have witnessed a substantial evolution in the treatment of chronic lymphocytic leukemia (CLL) that is the most common type of leukemia in Western societies. Immunochemotherapy with monoclonal antibody anti-CD20, rituximab combined with fludarabine and cyclophosphamide (FCR) has become the new standard first line therapy for younger and fit patients, after the results of CLL10 study by Hallek et al. [1] showed higher response rates and significantly longer overall survival (OS) in patients treated with FCR as compared with FC chemotherapy. However, CLL is a disease of the elderly, which means that most patients are less fit and have concomitant diseases. This makes them ineligible for the FCR protocol due to its toxicity. The group of CLL patients has been underrepresented in clinical trials with new agents for many years. The situation has changed recently. In 2014, the new monoclonal antibody obinutuzumab received European Medicine Agency (EMA) approval for the use in combination with chlorambucil as initial therapy of elderly, less fit CLL patients who are not qualified to intensive immunochemotherapy with FCR [2]. Obinutuzumab is a new humanized, glycoengineered type II IgG1 antibody against CD20 which has a different mechanism of action and enhanced efficacy in killing CLL cells in vitro compared to rituximab. The results of CLL11 study by Goede et al. [2] demonstrated that OS of patients treated with obinutuzumab-chlorambucil was significantly longer as compared to chlorambucil monotherapy.

Recent advances in understanding of CLL biology resulted in the development of the novel class of targeted therapies that have the potential to change the CLL treatment paradigm. In 2014, two oral tyrosine kinase inhibitors (TKI), ibrutinib (Bruton's kinase inhibitor) and idelalisib (phosphatidylinositol 3-kinase delta inhibitor) received EMA approval for the treatment of CLL patients who have received at least one prior therapy, or in first line in the presence of $17 \mathrm{p}$ deletion or TP53 mutation in patients unsuitable for chemo immunotherapy $[3,4]$. In CLL, $17 \mathrm{p}$ deletion/TP53 mutation correlates with unfavorable clinical course and extremely poor prognosis. TKI block signals from microenviroment essential for leukemic cells proliferation, differentiation and survival by inhibiting B-cell receptor (BCR) signaling. In phase II and III clinical trials ibrutinib has demonstrated tumor reduction and durable responses both in heavily pretreated patients as well as in patients with $17 \mathrm{p}$ deletion. Importantly, the drug is well tolerated in the elderly and less fit patients. Another promising class of targeting agents in CLL are Bcl-2 protein antagonists that induce apoptosis in leukemic cells. The most enhancing results have been demonstrated with ABT-199 that entered recently phase III clinical trials [5]. It has been shown to be effective in patients with ultra-high risk CLL, refractory to fludarabine or/and with $17 \mathrm{p}$ deletion. The landscape of CLL treatment is profoundly changing together with entering the era of targeted therapies, however whether CLL could be treated without chemotherapy and how to incorporate new agents into hitherto used cytotoxic drugs still remains an open question to be answered in ongoing and future clinical trials.

\section{Targeted therapy in chronic myeloid leukemia}

Chronic myeloid leukemia (CML) is a myeloproliferative neoplasm originating from primitive hematopoietic stem cells and characterized by the growth of myeloid cells in the bone marrow and accumulation of these cells in the blood. The specific genetic marker is Philadelphia chromosome and BCR-ABL fusion gene as a result of $\mathrm{t}(9 ; 22)(\mathrm{q} 34 ; \mathrm{q} 11)$. BCR-ABL protein has a tyrosine kinase activity and promotes leukemic growth [6].

The modern era of CML therapy began from introducing of imatinib - the first of tyrosine kinase BCR-ABL inhibitors (TKI). The IRIS (International Randomized Study of Interferon and STI571) trial demonstrated an 8-year survival for imatinib recipients of $85 \%$, but further studies showed that this drug was poorly tolerated in $\sim 20 \%$ and inadequate to achieve or maintain an optimal response also in $\sim 20 \%$. The mutations of BCR-ABL gene are the main cause of the poor responsiveness on imatinib. The novel TKI inhibitors - nilotinib, dasatinib and bosutinib overcome imatinib resistance, except T315I mutation. In the such clinical situation there are indications to allogeneic bone marrow transplantation [7].

In Department of Hematooncology and Bone Marrow Transplantation of the Independent Public Clinical Hospital No. 1 in Lublin, all modern forms of CML therapy are conducted. The decision about choosing the best therapy strategy is based on the guidelines released by European Leukemia Net (ELN) and Polish Group for Adult Leukemia (PALG). The special program of CML treatment, funded by National Health Service, provides very important support for clinicians. This program considers monitoring of cytogenetic and molecular response by using cytogenetics testing and RT-Q-PCR method. According to ELN guidelines BCRABL transcript level $>10 \%$ entitles us to change of imatinib therapy on TKI second generation [8]. Future directions for us will focus on the possibility of discontinuation of TKI therapy and potential cure of CML [9].

\section{Novel drugs in the treatment of multiple myeloma}

Multiple myeloma (MM), one of the most common hematological malignancies, is a clonal B-cell disease characterized by the proliferation of plasma cells in the bone marrow and typically accompanied by the secretion of monoclonal immunoglobulins. During the past decades, the paradigm of MM therapy has changed dramatically from the combination of oral melphalan+prednisone and high-dose chemotherapy with autologous stem cell transplantation (ASCT) for younger patients to the present regimens with the use of novel drugs such as proteasome inhibitor bortezomib and immunomodulatory drugs - thalidomide and its more potent derivative lenalidomide [10]. The new drugs to be used in combination with cytostatic therapy have been developed based on increased understanding of the biology of the disease like microenvironmental interactions between malignant plasma cells and the bone marrow [11]. Using them as a part of initial therapy is associated with high overall response rates as well as high rates of complete response, both for elderly and for younger patients treated prior to ASCT. 
In case of younger patients eligible for high-dose therapy and ASCT, bortezomib as well as thalidomide and lenalidomide tend to improve the ASCT results. For example, the combination of bortezomib and dexamethasone showed significantly better response rates when applied to conventional induction therapy prior ASCT [12].

In the group of older persons not eligible for ASCT, bortezomib has proven more effective than conventional chemotherapy. A multicenter VISTA trial was designed to compare the efficacy of melphalan+prednisone+bortezomib (MPV) regimen versus melphalan+prednisone (MP) in this group of patients [13]. The overall survival benefit with VMP versus MP was seen both overall and in an analysis restricted to patients who had received subsequent therapy [14]. MM patients invariably relapse after initial treatment strategies, so maintenance therapy to prolong response is of great importance. The use of novel drugs in this part of therapy seem to result in longer survival what is under investigation now.

Second generation proteasome inhibitors (carfilzomib) and immunomodulatory agents (pomalidomide) have recently been approved. Altogether, the introduction of novel agents for the treatment of MM patients has resulted in a 50\% improvement in median survival andactive preclinical and clinical research based on the knowledge of novel targets are still ongoing [10].

TABLE 1. APSCT performed in the Dept. of Hematooncology and Bone Marrow Transplantation in Lublin.

\begin{tabular}{lcc}
\hline \hline \multicolumn{1}{c}{ APSCT indication } & APSCT 1997-2004 & $\begin{array}{c}\text { APSCT 2005-2014 } \\
\text { (including July 2014) }\end{array}$ \\
\hline $\begin{array}{l}\text { Multiple myeoma/ } \\
\text { Amyloidosis/Z.POEMS }\end{array}$ & 102 & 329 \\
\hline Hodgkin`sdisease & 35 & 96 \\
\hline Non-Hodgkin`s lymphoma & 39 & 87 \\
\hline Acute myeloid leukemia & 9 & 23 \\
\hline Acute lymphoid leukemia & 12 & 3 \\
\hline Chronic myeloid leukemia & 2 & 1 \\
\hline Ca mammae/Ca testis & $3 / 2$ & $0 / 4$ \\
\hline Total & $\mathbf{2 0 4}$ & $\mathbf{5 4 3}$ \\
\hline
\end{tabular}

TABLE 2. AlloBMT performed in the Dept. of Hematooncology and Bone Marrow Transplantation in Lublin.

\begin{tabular}{|c|c|c|c|c|c|c|}
\hline Year & $\begin{array}{c}\text { Acute } \\
\text { myeloid } \\
\text { leukemia }\end{array}$ & $\begin{array}{c}\text { Acute } \\
\text { lymphoid } \\
\text { leukemia }\end{array}$ & $\begin{array}{c}\text { Chronic } \\
\text { myeloid } \\
\text { leukemia }\end{array}$ & $\begin{array}{l}\text { Hodgkin`s } \\
\text { disease }\end{array}$ & $\begin{array}{l}\text { Aplastic } \\
\text { anaemia }\end{array}$ & Total \\
\hline 2005 & 1 & 1 & & & 1 & 2 \\
\hline 2006 & 2 & 3 & & & & 5 \\
\hline 2007 & 2 & 2 & & & & 4 \\
\hline 2008 & 1 & 2 & & & & 3 \\
\hline 2009 & & 4 & & & & 4 \\
\hline 2010 & 2 & 1 & & & & 3 \\
\hline 2011 & 2 & 2 & & & & 4 \\
\hline 2012 & 3 & 1 & & & & 4 \\
\hline 2013 & 1 & 1 & 1 & & & 3 \\
\hline 2014 & 2 & 2 & & & & 4 \\
\hline Total & 16 & 19 & 1 & & 1 & 37 \\
\hline
\end{tabular}

\section{Bone marrow transplantation procedures in hematologi- cal disorders}

Bone Marrow Transplantation Unit is a part of the Department of Hematooncology and Bone Marrow Transplantation of Medical University in Lublin. It is the only transplantation unit for adults in Lublin area. We started with autologous transplantation of peripheral stem cells (APSCT) in Nov 1997. The first successfully transplanted patient with multiple myeloma lived till 2004. In that time, the Unit was situated in the Clinic Hospital No 4 in Lublin. It consisted of 4 rooms for transplantation. Until 2004, we performed 204 APSCT in patients with different diseases - indications are summarized in Table 1. In 2004, we moved to a new transplantation unit built in the Clinic Hospital No 1. The new unit consisted of 9 rooms with 12 beds for autologous and allogeneic transplantation (alloBMT). We started with allogeneic transplantation from sibling donors in 2005 in a patient with acute lymphoblastic leukemia. We also continued to perform APSCT. Between 2004 and 2014 we performed 543 APSCT - the indications are summarized in Table 1 and 37 alloBMT - indications are summarized in Table 2.

During the last decade, there were some huge advances in transplantation procedures $[15,16]$. It refers to either mobilization procedures which are performed with the use of effective protocols with high dose of etoposide or cytarabine, reinduction chemotherapy and plerixafor or efficacious treatment of the patients before transplantation with new drugs e.g. bortezomib, rituximab thalidomide, lenalidomide, imatinib, busilvex. During transplantation procedure we can offer better supportive care using new antibiotics, antifungal agents, antiviral, cytoprotective drugs and after transplantation, if indicated maintenance treatment.

\section{REFERENCES}

1. Hallek M, Fischer K, Fingerle-Rowson G, et al. Addition of rituximab to fludarabine and cyclophosphamide in patients with chronic lymphocytic leukaemia: a randomized, open-label, phase 3 trial. Lancet. 2010;376:1164-74.

2. Goede V, Fischer K, Busch R, et al. Obinutuzumab plus chlorambucil in patients with CLL and coexisting conditions. N Engl J Med. 2014;370:1101-10.

3. Martyr S, Aue G, Saba N, et al. Ibrutinib for previously untreated and relapsed or refractory chronic lymphocytic leukaemia with TP53 aberrations: a phase 2, single-arm trial. Lancet Oncol. 2015;16:169-76.

4. Burke RT, Meadows S, Loriaux MM, et al. A potential therapeutic strategy for chronic lymphocytic leukemia by combining Idelalisib and GS-9973, a novel spleen tyrosine kinase (Syk) inhibitor. Oncotarget. 2014;28:908-15.

5. Souers AJ, Leverson JD, Boghaert ER, et al. ABT-199, a potent and selective BCL-2 inhibitor, achieves antitumor activity while sparing platelets. Nat Med. 2013;19:202-8.

6. Jabbour AJ, Kanterjian H. Chronic myeloid leukemia: 2014 update on diagnosis, monitoring, and management. Am J Hematol. 2014;89:54856.

7. Hughes T, White D. Which TKI? An embarrassment of riches for chronic myeloid leukemia patients. Hematology Am Soc Hematol Educ Program. 2013;2013:168-75.

8. Baccarani M, Deininger MW, Rosti G, et al. European Leukemia Net recommendations for the management of chronic myeloid leukemia. Blood. 2013;122:872-84.

9. Sweet K, Oehler V. Discontinuation of tyrosine kinase inhibitors in chronic myeloid leukemia: when is this a safe option to consider? Hematology Am Soc Hematol Educ Program. 2013;2013:184-8. 
10. Spicka I. Advances in multiple myeloma therapy during two past decades. CSBJ. 2014;10:38-40.

11. Röllig C, Knop S, Bornhäuser M. Multiple myeloma. Lancet.doi: 10.1016/S0140-6736(14)60493-1 [Epub ahead of print].

12. Scheid C, Sonneveld P, Schmidt-Wolf IG, et al. Bortezomib before and after autologous stem cell transplantation overcomes the negative prognostic impact of renal impairment in newly diagnosed multiple myeloma: a subgroup analysis from the HOVON-65/GMMG-HD4 trial. Hematol. 2014;99:148-54.

13. San Miguel JF, Schlag R, Khuageva NK, et al. VISTA trial investigators: bortezomib plus melphalan and prednisone for initial treatment of multiple myeloma. N Engl J Med. 2008;359:906-17.

14. Harousseau JL, Palumbo A, Richardson P, et al. Superior outcomes associated with complete response in newly diagnosed multiple myeloma patients treated with non-intensive therapy: analysis of the phase 3 VISTA study of bortezomib plus melphalan-prednisone versus melphalanprednisone. Blood. 2010;116(19):3743-50.

15. Hopman RK, DiPersio JF. Advances in stem cell mobilization. Blood Rev. 2014;28:31-40

16. Arnaout K, Patel N, Jain M, et al. Complications of allogeneic hematopoietic stem cell transplantation. Cancer Invest. 2014;32:349-62.

\section{Corresponding author}

Marek Hus

Department and Bone Marrow Transplantation, Medical University of Lublin 11 Staszica Str., 20-081 Lublin

E-mail: markhus@go2.pl 